\title{
APPLICATION OF UAV OBLIQUE PHOTOGRAPHY IN REAL SCENE 3D MODELING
}

\author{
Zhou Tihui ${ }^{1}$, Lv Linbing ${ }^{1}$, Liu Jinhu ${ }^{1}$, Wan $\mathrm{Ji}^{2}$ \\ ${ }^{1}$ National Quality Inspection and Testing Center for Surveying and Mapping Products, Beijing, China - 442002023@qq.com \\ ${ }^{2}$ Hunan Surveying and Mapping Products Quality Supervision and Inspection Station, Changsha, China - 306488692@qq.com
}

KEY WORDS: UAV, Oblique Photography, 3D Model, Triangulation, Plane Accuracy, Elevation Accuracy.

\begin{abstract}
:
Aiming at the phenomenon that the traditional measurement methods cannot complete large-scale measurement in a short time, and the image quality obtained by remote sensing in cloudy and rainy areas is difficult to meet the demand, this paper puts forward the idea of using UAV tilt photography to build three-dimensional modeling of urban real scene. The UAV tilt photography technology is used to collect the image data of about $200 \mathrm{~km} 2$ in Wuzhishan City. By laying a small number of ground image control points, the aerial triangulation is used to establish the connection, and the three-dimensional modeling of the collected data is carried out. Through the field verification to verify the attribute information of ground objects, the accuracy is verified by using CORS system field coordinate collection. The results show that the ground property and mathematical accuracy of UAV tilt photography model meet the requirements, and can be effectively used in real 3D modeling.
\end{abstract}

\section{INTRODUCTION}

UAV appeared in the early 20th century. Early UAV were mainly used in the military field for combat, reconnaissance and civilian remote sensing. Entering the 21 st century, with the development of computer technology and communication technology and the advent of various hardware sensors, the performance of drones has been continuously improved, the scope of application and fields have been continuously expanded, and the endurance and load weight have been greatly improved (Li D., Li Ming, 2014; Bi Kai, 2015). In recent years, the drone tilt photogrammetry technology developed by the integration of drones and tilt photogrammetry has changed the limitation that traditional aerial survey and remote sensing can only be taken from the vertical direction, using multiple sensors to capture data from different angles. Collect, efficiently and quickly obtain massive amounts of data and information, and truly and reliably reflect the objective conditions on the ground. Because of these technical characteristics, UAV oblique photogrammetry technology has begun to be gradually applied in $3 \mathrm{D}$ modeling.

HereMap has released high-resolution oblique photographic 3D city models of Berlin, Milan, Madrid, Los Angeles and other cities around the world. All oblique models released by it are reconstructed from oblique images collected by low-altitude aircraft including drones. The oblique model is divided into uniform blocks, the detail-level model is established and published to the Internet, so that users can browse the real 3D model of the city online. Many cities such as Beijing and Shanghai in China have carried out researches and model productions of urban reality 3D construvtion for visualization, urban space planning, and decision-making assistance (Zhou X., et al., 2016; Liao Y. et al., 2019; Chu C., 2020; Li L., Guo Z., Zhang Q.,2020).

Compared with traditional measurement techniques, UAV oblique photogrammetry has obvious advantages, mainly as follows: (1) Its flying height is highly flexible and suitable for aerial photography missions in many different regions and terrains. The flying height of the UAV can be from several meters to several kilometers, and can be set freely according to the mission requirements. (2) It has rich sources of images and is of high overlap. The UAV can be equipped with multi-lens cameras, and the multi-angle camera group can obtain the top surface and side image data of the ground objects in multiple directions and high coverage.(3) It has a high degree of automation. Set the corresponding parameters before reclining the drone, and the drone can automatically fly according to the corresponding settings. The main process of automatic image matching and modeling is completed by the computer. (4) The side texture of the solid is visible. The traditional digital orthophoto map mainly obtains the top texture of the entity, while the oblique photography technology can simultaneously obtain rich mapping side textures to truly show the true shape of the ground object. (5) It is cost-effective. UAV oblique photogrammetry technology has higher efficiency in data collection and city $3 \mathrm{D}$ model production, which can reduce time and labor costs.

\section{THE KEY TECHNOLOGY OF DRONE OBLIQUE PHOTOGRAMMETRY}

\subsection{Fundamental}

The basic principle of oblique photogrammetry is a process of data collection by the oblique camera system, which carries multiple sensors, generally composed of high-resolution cameras with a vertical angle in the middle and 4 oblique angles around it, from 5 directions at the same time, high-resolution aerial images are collected, and the positive and oblique films of different cameras are superimposed together to form a twodegree or three-degree overlap with the ground points to form an oblique photogrammetric image covering the survey area. The geometric spatial posture of the oblique piece, combined with ground control points, realizes 3D modeling of ground objects. Fig. 1 shows the workflow of the real 3D model of oblique photogrametry. 


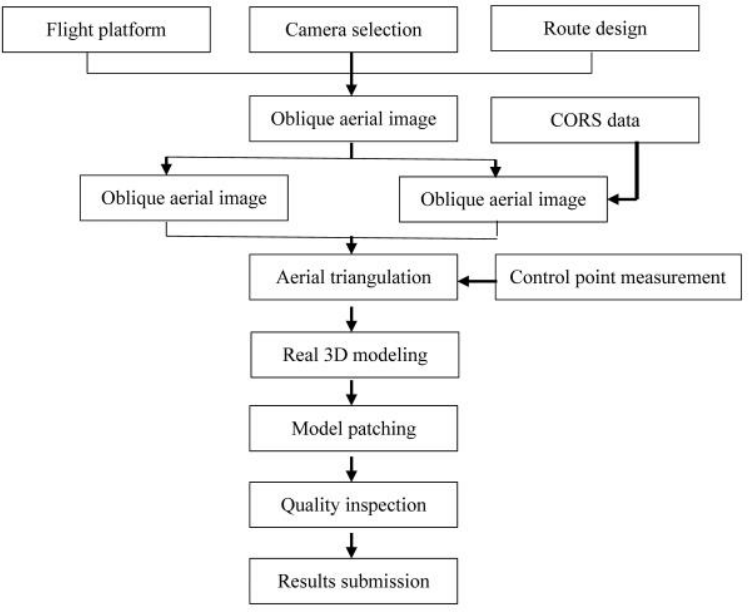

Figure 1. Workflow of the oblique photogrametry real 3D model processing

\subsection{Aerial Photography Platform Selection and Route Design}

UAV can be divided into fixed wing and rotary wing, which are suitable for operation at different flying heights. In practical, the selection of a suitable drone depends on the conditions of the survey area and the types of aerial camera. For the same oblique camera, the higher the flying altitude, the lower the image resolution. To achieve project results and meet the needs of users for actual effects, it is crucial to applythe most suitableoblique camera. The current high-precision oblique aerial camera systems on the market include SWDC-5AP100 from 4D Vision, AMC5100 from Shanghai Hangyao Company, and RCD30 Oblique from Leica, etc. Among them, AMC5100 has a single lens of 100 million pixels and a 5-lens with a total of 500 million pixels that shows good image quality.

The first thing that needs to be determined in route design is the flight altitude, shown in equation (1).

$$
h=\frac{f * G S D}{a}
$$

where $\quad h=$ relative altitude

$f=$ lens focal length

$a=$ Pixel size

$G S D=$ ground resolution

High image resolution and overlap are the key factors that determine the accuracy of the results, while a reasonable route scheme is the critical part to achieve the goal. In practical, it should be designed according to the requirements of aerial photography projects and the actual geomorphic characteristics of the survey area, and the route direction should be arranged according to the survey area. For route laying of oblique photography, the overlap of images generally requires a minimum of $70 \%$ end and side overlap (Gu G. et al., 2019). To ensure the effect in densely-built areas, increase the overlap appropriately when laying routes degree.

\subsection{Image Control Point Layout}

The control measurement is to ensure the accuracy of the aerial three and determine the absolute position of the ground object in the space. In the conventional low-altitude digital aerial photogrammetry field specifications, there are detailed regulations on the layout of control points, which are the basis for ensuring accuracy. Compared with traditional photography technology, oblique photography requires a higher degree of image overlap. By increasing the placement density of image control points, the accuracy of the $3 \mathrm{D}$ model results can be improved. Under the condition of meeting the accuracy requirements of the model, multiple factors are combined to select the optimal image control point layout plan. Under the premise of ensuring the spatial accuracy of the results of the $3 \mathrm{D}$ model, the image control points are usually evenly distributed in each zone. At least one image control point is arranged in a flat area $1 \mathrm{~km} 2$, and the image control points are appropriately encrypted at the edge of the zone (Liu Z., 2019).

\subsection{Oblique Aerial Triangulation}

Oblique photography aerial triangulation mainly uses POSassisted aerial triangulation. The steps include connection point extraction and beam method regional network adjustment. The idea of extracting connection points of oblique images is to use the external orientation elements of the image provided by the POS system as the initial value, and use the scale and affine invariant feature algorithm to perform feature matching of multi-view images to obtain the connection points between the images. The beam method area network adjustment is the mainstream method of aerial triangulation, with strict algorithm theory and high encryption accuracy. This method is also suitable for aerial triangulation of multi-view images.

\subsection{D Model Construction}

After the aerial triangulation is passed, the 3D modeling is performed. The modeling software automatically matches the points with the same name in the image according to the image matching algorithm, extracts the feature points to form a dense point cloud, builds a triangular TIN from the feature points, and then a white model from the triangular TIN. Calculate the corresponding texture from the image, and automatically map the texture to the corresponding white mold to complete the real 3D modeling process. The whole process is automated by the oblique 3D modeling system software.

\section{APPLICATION CASE AND ANALYSIS}

\subsection{Applications Case}

Wuzhishan City is selected as an example for application analysis. The project covers an area of about $200 \mathrm{~km}^{2}$. Wuzhishan City is located in the hinterland of Wuzhishan in the central and southern part of Hainan Island, and its geographical coordinates are $109^{\circ} 19^{\prime}-109^{\circ} 44^{\prime} \mathrm{E}$ and $18^{\circ} 38^{\prime}-19^{\circ} 02^{\prime} \mathrm{N}$. The survey area belongs to subtropical climate, cloud and rain weather is frequent, so it is not suitable for carrying out largescale aerial photogrammetry. The flexible and mobile performance of tilt photography of UAV is suitable for completing the task of this project. The project requires that the image resolution should be no less than $5 \mathrm{~cm}$, and the production of large scene 3D model should be made to provide technical support and data support for the modernization development of the city. The terrain in the survey area is complex, and before aerial photography, the terrain, landform and airspace within the survey area should be considered comprehensively, and the actual situation of the survey area should be combined to conduct zonal aerial photography, so as to meet the requirements of the achievement indicators. Due to more cloud and rain weather and large area in the test area, the project adopts a combination of fixed wing and rotor wing to meet the 
requirements of the project. Fixed wing Locust 3.0 UAV and six-rotor SH-20X UAV are mainly adopted, as shown in Figure 2 and Figure 3.

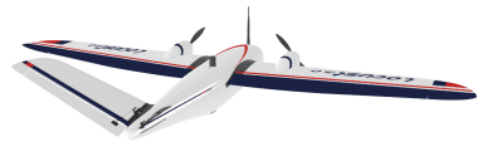

Figure 2. Fixed wing locust 3.0 UAV

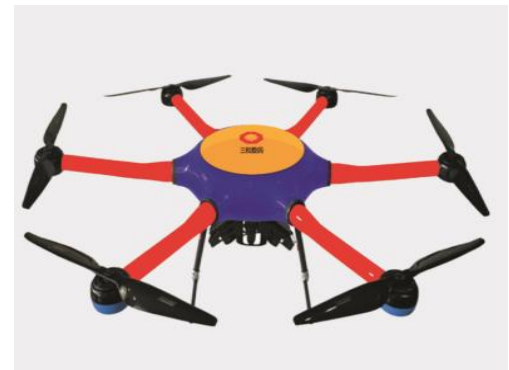

Figure 3. Six-rotor sh-20x UAV

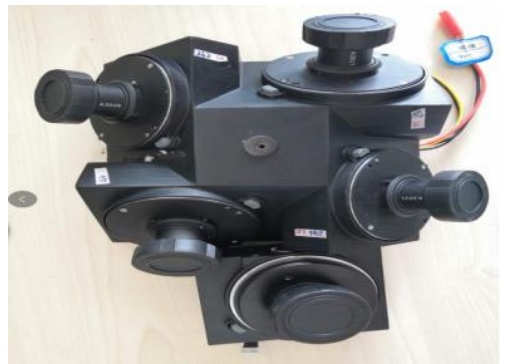

Figure 4. Five angle of view oblique photography lens

The aerial camera adopts the five lenses of Lingjing A5, as shown in Figure 4. Lingjing A5 five lens tilt photography aerial camera is a special equipment for 3D modeling data acquisition, with a total pixel of 120 million. A single camera uses 24.3 million pixels APS-C frame CMOS sensor, BIONZ X-ray image processor, which supports regional noise reduction, has high low sense definition and strong ability of noise control with high sense. Combined with $20 \mathrm{~mm}$ (orthophoto) and $35 \mathrm{~mm}$ (lateral) professional aviation mechanical lens, the image processing ability is further improved. Small size, light weight; with trigger module, it supports flight control trigger at the same time; with fast mounting design, it is easy to install and suitable for various UAV platforms. The main parameters of Lingjing A5 five lens tilt aerial camera are shown in Table 1.

\begin{tabular}{|l|l|}
\hline CCD number & $5 \mathrm{pcs}$ \\
\hline CCD size & $23.5 \mathrm{~mm} \times 15.6 \mathrm{~mm}$ \\
\hline Total pixels & 120 million \\
\hline Single effective pixel & 24 million 300 thousand \\
\hline focal length & Orthophoto $20 \mathrm{~mm}$, tilt $35 \mathrm{~mm}$ \\
\hline Pixel physical size & $3.9 \mu \mathrm{m}$ \\
\hline Image resolution & Better than $2 \mathrm{~cm}$ \\
\hline Lens tilt angle & $40^{\circ}$ \\
\hline
\end{tabular}

Table1. Main parameters of Lingjing A5 aerial camera

In order to achieve the resolution of $5 \mathrm{~cm}$ and aerial photography overlap requirements, this design has a certain amount of surplus to ensure that the project needs to meet. The ground resolution meter on the datum is $0.045 \mathrm{~m}$, the course overlap is $80 \%$, and the side overlap is $75 \%$. In the densely built-up area, the degree of overlap should be appropriately increased.

Due to the scattered distribution of the photography area, part of the photography area is small. In order to meet the subsequent production needs, 8 10 image control points are laid per square kilometer, among which 2 points are used as checkpoints. The image control points are evenly distributed, and there are control points at the junction of flight flights. The signs of the image control points are shown in Figure 5. The image control points should be located where the terrain is flat and there are obvious markers. The oblique photography digital aerial photography route is laid according to the conventional method. The lateral coverage needs to guarantee the threedimensional modeling of the camera area boundary, and the course coverage needs to guarantee the three-dimensional modeling of the camera area boundary, which generally exceeds the camera area boundary by no less than four baselines.

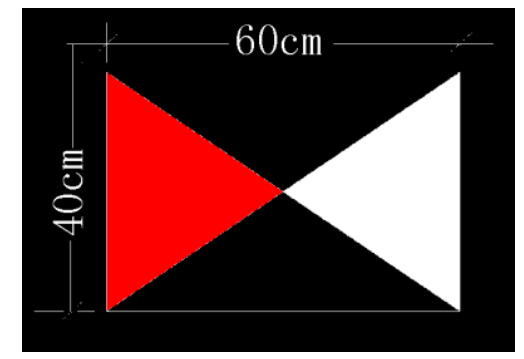

Figure 5. Image control point layout sign

Absolute loopholes, relative loopholes and other serious defects in aerial photography must be corrected in time. Repair must be carried out according to the original design track. The length of the supplementary photography route should meet the requirements of the encryption distribution points of the user area network. For the relative loopholes and local defects that do not affect the internal encryption selection points and model connection, such as clouds, cloud shadows, speckle, etc., the supplementary shots can be taken only on the loopholes. The length of the supplementary shooting route should exceed four baselines outside the leak. A tilt digital aerial camera with the same main distance and type should be used for supplementary photography. A minimum of 7 images should be overlapped during tilt photography. When using POS assisted oblique aerial photography, it should be carried out in accordance with the corresponding supplementary and re-photographing requirements. There will be tonal differences between the oblique image and the nadir image, so it is necessary to conduct batch light and color levelling processing for the image, as shown in Fig. 6.

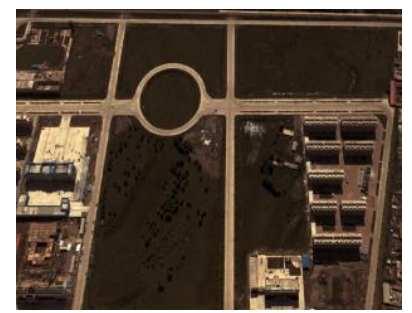

(a)

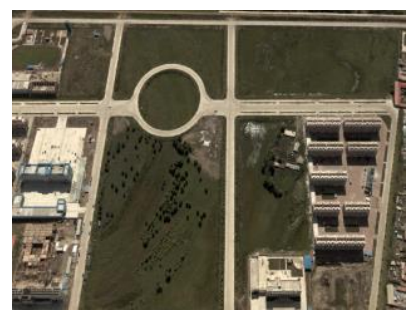

(b)
Figure 6. Contrast the image(a) before and (b) after saturation balance 
The 3D modeling software has been able to realize automatic modeling. The 3D modeling of this project adopts the Smart3D software, which will automatically select the best image pair model in the opposite perspective according to the internal rule algorithm, and finally form the three-dimensional scale dense point cloud. The point cloud is then automatically converted to the irregular triangulation TIN structure, and the TIN caused by image matching errors is deleted and repaired. Based on the inherent geometric relationship, the TIN model is smoothed and optimized to achieve the best three-dimensional expression effect. Finally, according to the spatial location information of 3D TIN, the optimal perspective image is automatically found, and the texture matching construction of the model is completed Finally, a complete and real 3D model is formed after manual modification and editing. The specific 3D modeling process includes the following four steps.

The first step is to generate point cloud-pairs. Based on the results of the external orientation elements output by the null tri-adjustment and the relationship between the camera placement position, Smart3D software can automatically find the appropriate two images to form a 3D image pair. Then the point cloud matching calculation is carried out for the $3 \mathrm{D}$ image pairs.

The second step is to construct a three-dimensional TIN network. The obtained point cloud is triangulated by the algorithm, and the triangulation diagram of a certain region is shown in Fig 7 During this process, some abnormal points are discarded as rough handicap because normal triangles cannot be constructed.

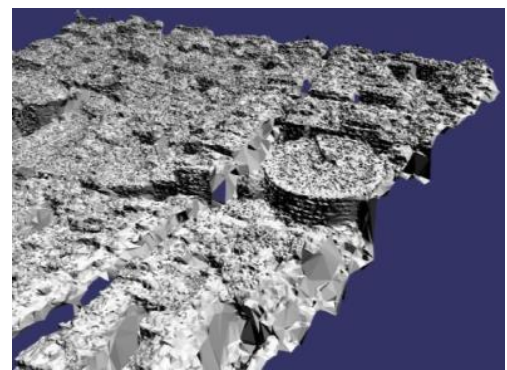

Fig 7. TIN network constructed by point cloud

The third step is the optimization of 3D TIN network. Smart3D software is embedded with a variety of advanced intelligent algorithms, which can automatically detect and evaluate irregular triangulation network, automatically optimize unreasonable triangulation network surface, automatically simplify and sparse the triangular density on flat surface, and retain the triangular density on complex surface. The optimized 3D TIN is shown in Figure 8.

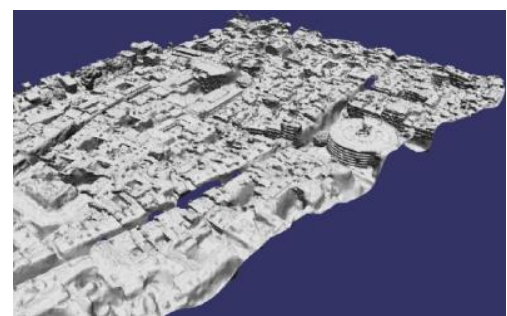

Fig 8. Optimized 3D TIN network
The fourth step is texture matching. Smart3D software can automatically map the image of the best perspective as the model texture according to the spatial position of each triangle in the TIN network, as shown in Figure 9.

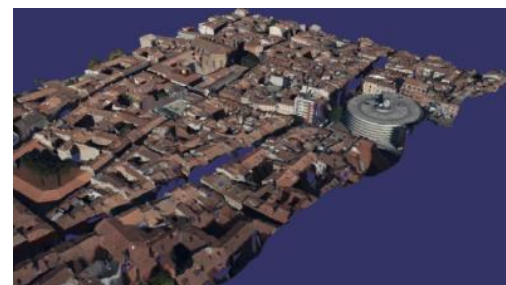

Fig 9. The diagram of 3D schematic after texture mapping

\subsection{Products Analysis and Accuracy Verification}

The overall effect of the 3D model constructed by tilt photography is good. Based on the viewing platform of the $3 \mathrm{D}$ model, the 3D model can be zoomed to the field of view that matches the actual resolution of the image, and the true 3D model results can be viewed by 360 degrees rotation. No large area of the model is redundant or omitted in the scene.

In the vegetation area, the tone of the vegetation area is basically the same as the real scene, as shown in Figure 10. The boundary of different vegetation is clear, and the ridge of the field is clear, which can meet the multi-functional purposes such as land and vegetation survey in rural areas.

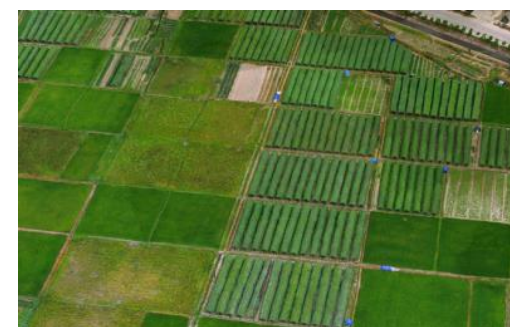

Fig 10. 3D visualization of vegetation area

In rural residential areas, the vegetation and houses are basically the same as the real scene, and houses and vegetation are clearly distinguished, as shown in Figure 11. Due to the technical characteristics of tilt photography, the junction between the house and the tall trees is partially shaded.

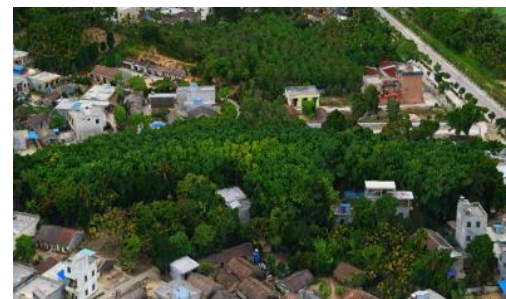

Figure 11.3D visualization of Rural residential area

In urban areas, there are a large number of high-rise building, the plane will produce large amounts of the ownership information overlapping and redundancy, three-dimensional system can be vast amounts of data on the space for precise positioning, display, intuitively showing position and attribute information, better suited to the demands of the development of modern city information figure 12 for a 3D rendering of real city. As can be seen from Figure 12 at the beginning of the analysis, the geographical elements of the three-dimensional 
model are complete in types, with clear dividing lines between various ground features and seamless connection between geographical elements. The texture, color, shape, spatial position and relative relationship of the ground objects are consistent with the actual environment; Building, site and other main features of the main body integrity.

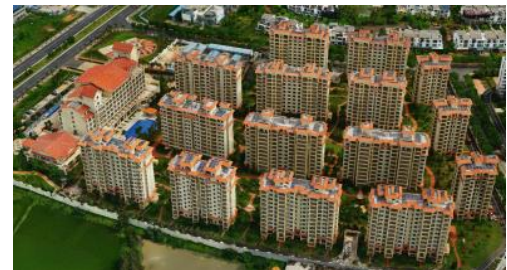

Fig 12.3D visualisation of urban reality 3D model

The overall quality of 3D modeling results is good, but there are still some problems in a few areas, which need to be further improved. Buildings covered by trees in densely vegetated areas are not well shown and need to be supplemented by other means of mapping. In the surface of the water, there are loopholes, uneven color and other phenomena, a large range of voids can be processed automatically by software, part of the 3D model needs to be manually edited and improved. Fig. 13 is before and after editing the three-dimensional void on the water surface, Fig. 14 is before and after editing the uneven tone, and Fig. 15 is before and after editing the suspended objects in the three-dimensional scene. Other quality problems need to be modified and improved according to the actual situation.

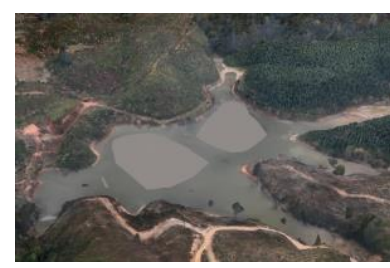

(a)

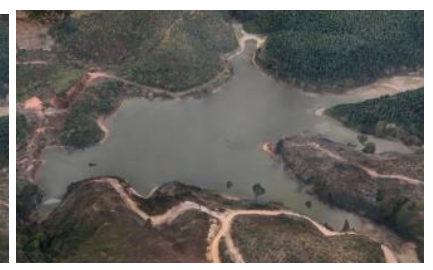

(b)
Figure 13. The effect of the restoration of the holes in water (a) before, (b) after.

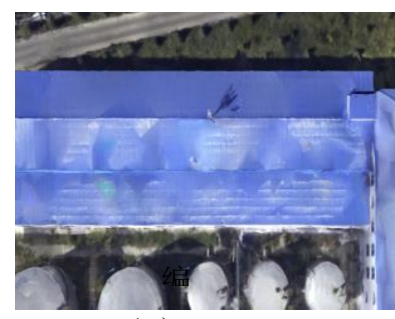

(a)

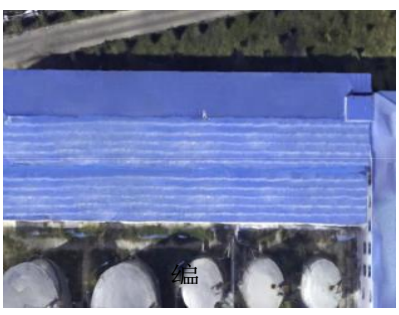

(b)
Figure 14. The effect of the restoration of uneven color on the roof (a) before, (b) after.

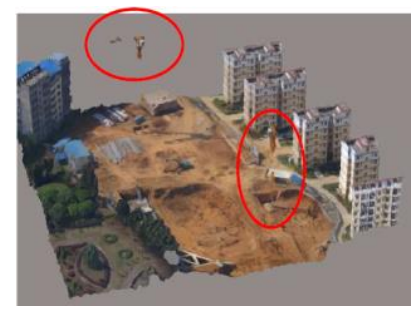

(a)

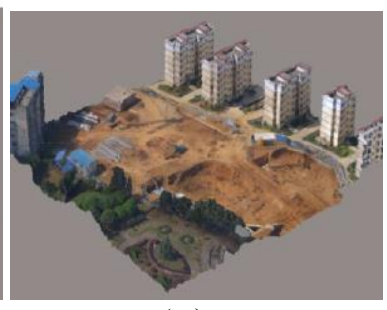

(b)
Fig 15. Editing of suspended objects(a) before, (b) after.
The accuracy of this project is carried out by means of field accuracy verification. The following takes a certain area in Wuzhishan City as an example. Use RTK to receive signals from the Hainan CORS station to collect the ground plane and elevation coordinates, and compare them with the coordinate information of the points of the same name in the threedimensional model. Among them, the plane coordinate collection compares 95 points and the elevation compares 24 points. Specific statistics are shown in Table 2.

\begin{tabular}{|c|c|c|c|c|}
\hline type & $\begin{array}{c}\text { Max } \\
/ \mathrm{m}\end{array}$ & $\begin{array}{c}\text { Min } \\
/ \mathrm{m}\end{array}$ & $\begin{array}{c}\text { average } \\
/ \mathrm{m}\end{array}$ & $\begin{array}{c}\text { Standard } \\
\text { error } \\
/ \mathrm{m}\end{array}$ \\
\hline $\begin{array}{c}\text { plane } \\
\text { accuracy }\end{array}$ & 0.252 & 0.013 & 0.085 & 0.102 \\
\hline $\begin{array}{c}\text { elevation } \\
\text { accuracy }\end{array}$ & 0.275 & 0.028 & 0.098 & 0.124 \\
\hline
\end{tabular}

Table 2. Statistics of plane and elevation accuracy

It can be concluded from Table 1 that the error in the plane of the $3 \mathrm{D}$ model is $0.102 \mathrm{~m}$, and the error in elevation is $0.124 \mathrm{~m}$, which can meet the 1:500 mapping and project requirements.

\section{CONCLUSIONS}

This paper takes the application of 3D modeling of UAV oblique photography technology as an example, and analyzes the results, and conducts field inspections and accuracy verification of the results. The application results show that the result data can meet the requirements of 3D building multiple geographic information attributes as a whole, and the plane and elevation accuracy of the 3D model meets the accuracy requirements of surveying and modeling, but there are still a few quality defects after automated modeling, and subsequent corresponding processing is required edit. Compared with traditional technical methods, the method of using drone oblique photography to construct a 3D model is efficient, fast and collects rich information, which is convenient for subsequent statistical analysis and will play an important role in future surveying and mapping geographic information data collection.

\section{REFERENCES}

Bi K., Li Y., Ding X., 2015. The Status and Development Trend of Aerial Photography Technology of Light and Small UAV. Bulletin of Surveying and Mapping, 3, pp.27-31.

Chu C., 2020. Auxiliary application of UAV oblique Photogrammetry in planning completion survey. Surveying and Spatial Geographic Information, 43(04), pp.205-208.

Gu G., Zhang K., Liu Z., 2017. Discussion on technical standard of UAV oblique Photogrammetry. Bulletin of Surveying and Mapping, S1, pp.210-213.

Li L., Guo Z., Zhang Q.,2020. Application of UAV Oblique Photogrammetry Technology in Urban Basic Surveying and 
Mapping. Surveying and mapping geographic information, 45(06), pp.72-74.

Li D., Li M., 2014. Research Progress and Application Prospects of UAV Remote Sensing System. Journal of Wuhan University (Information Science Edition), 39(05), pp.505-513.

Liao Y., Hu Y., Ye T., 2019. Application of low-altitude drone oblique photography in land management. Surveying and Spatial Geographic Information, 42(02), pp.97-100.

Liu Z., 2019. Research and Practice of Large-scale City Real Scene 3D Modeling Technology Based on Oblique Photography. Surveying and Spatial Geographic Information, 42(02), pp.187-189.

Zhou X., Meng X., Zhang X., 2016. Method for constructing true 3D model of city based on oblique photogrammetry. Surveying and Mapping Science, 41(09), pp. 159-163. 